\title{
Evaluation of the BD Phoenix Automated Microbiology System for Identification and Antimicrobial Susceptibility Testing of Common Clinical Isolates
}

\author{
Z.K. Liu T.K. Ling A.F. Cheng \\ Department of Microbiology, The Chinese University of Hong Kong, Prince of Wales Hospital, \\ Hong Kong, China
}

\section{Key Words}

BD Phoenix Automated Microbiology System · API

system $\cdot$ Microbroth dilution method

\begin{abstract}
Objective: To evaluate the accuracy and reliability of the BD Phoenix Automated Microbiology System for identification (ID) and antimicrobial susceptibility testing (AST) of Gram-positive and Gram-negative isolates. Materials and Methods: The ID of 291 Gram-negative and 158 Gram-positive clinical isolates were evaluated by the system. The AST of 252 Gram-negative and $151 \mathrm{Gram}$ positive isolates with correct ID were then evaluated. The results were compared with those of the API ID system and the microbroth dilution method. All discrepant results were repeated for verification. Results: Over $90 \%$ Gram-negative and Gram-positive isolates were correctly identified to species or genus level by the Phoenix system. The minimum inhibitory concentration agreement rates between the Phonenix and the microbroth dilution methods within \pm 1 dilution ranged from 87.3 to $97.6 \%$ for Gram-negative isolates, and from 50 to $100 \%$ for Gram-positive isolates. For Gram-positive isolates, vancomycin had significant low minimum inhibitory
\end{abstract}

concentration agreement rates. Conclusions: Overall, the results indicate that the Phoenix system is a reliable system. It could provide accurate ID and AST results for routine clinical laboratories.

Copyright (C) 2005 S. Karger AG, Basel

\section{Introduction}

Most routine microbiological tests are performed manually, although some automatic systems have been commercialized for over the last two decades [1-3]. The automatic systems will continue to develop because the laboratories are taking heavier workloads with limited resources. Two latest automation systems, the Phoenix Automated Microbiology System (Becton Dickinson, Sparks, Md., USA) and the Vitek 2 System (bioMérieux) are available on the market. More field evaluations of these systems are required before the conventional manual methods can be replaced [4-8]. Hence, the aim of this study was to assess the performance of the Phoenix Automated Microbiology System by comparing its results with those of the conventional methods for identification (ID) (the API ID system) and antimicrobial susceptibility testing (AST) (the microbroth dilution, MB, method).

\begin{tabular}{ll}
\hline KARGER & ( ) 2005 S. Karger AG, Basel \\
1011-7571/05/0144-0250\$22.00/0 \\
$\begin{array}{l}\text { Fax +4161306 } 1234 \\
\begin{array}{l}\text { E-Mail karger@karger.ch } \\
\text { www.karger.com }\end{array}\end{array}$ & $\begin{array}{l}\text { Accessible online at: } \\
\text { www.karger.com/mpp }\end{array}$
\end{tabular}

Dr. Thomas K.W. Ling

Department of Microbiology, The Chinese University of Hong Kong Prince of Wales Hospital

Hong Kong (China)

Tel. +852 26322307, Fax +852 26451256, E-Mail lingt@cuhk.edu.hk 


\section{Materials and Methods}

\section{Bacterial Strains}

Clinical isolates collected between 2000 and 2001 in the Prince of Wales Hospital, Hong Kong, were stored in cryotubes at $-70^{\circ} \mathrm{C}$. Different numbers of Gram-negative and Gram-positive strains were selected to set up a bacterial profile that reflected the projected ratio of different isolates in our routine clinical laboratory; 291 Gram-negative strains and 158 Gram-positive cocci were selected.

\section{The Inoculating Procedures of the Phoenix System}

Two hundred and ninety-one Gram-negative strains and 158 Gram-positive cocci were tested using the NMIC/ID-4 and the PMIC/ID-14 panels of the Phoenix system, respectively. Firstly, CrystalSPec nephelometer (Becton Dickinson, Sparks, Md., USA) was used to prepare the McFarland 0.5 bacterial suspension for the Phoenix ID tests in $4.5 \mathrm{ml}$ of Phoenix ID broth. Then, the bacterial suspension for the Phoenix AST test was prepared by adding $25 \mu l$ of bacterial ID suspension and one drop of AST indicator to $8 \mathrm{ml}$ of Phoenix AST broth. The prepared bacterial ID and AST suspension was poured into the ID and AST sector of the Phoenix panels, respectively. Each biochemical reaction well on the panels was rehydrated with $50 \mu \mathrm{l}$ of bacterial suspension. Excess suspension was collected by the absorbent pad at the bottom of the panels. After sealing with a plastic cover and scanning the panel barcode, the panel was loaded manually into the Phoenix system.

\section{Analysis of the ID Testing Results of the Phoenix System}

The ID results of 291 Gram-negative strains and 158 Grampositive cocci of the Phoenix system were compared with those of the conventional API system as references. Afterwards, the correct ID rates to genus and species levels were calculated.

\section{Analysis of the AST Results of the Phoenix System}

Gram-negative strains were tested by the Phoenix system against 13 antimicrobial agents including amikacin (Bristol-Myers Squibb), ampicillin-sulbactam (Pfizer), cefepime (Bristol-Myers Squibb), cefotaxime (Aventis), ceftazidime (GSK), chloramphenicol (Sigma), ciprofloxacin (Bayer), gentamicin (Sigma), imipenem (MSD), meropenem (Astra Zeneca), moxifloxacin (Bayer), piperacillin (Wyeth) and piperacillin-tazobactam (Wyeth). Gram-positive cocci including Staphylococcus spp. and Enterococcus spp. were tested against eight antimicrobial agents including ampicillin (Sigma), chloramphenicol, ciprofloxacin, erythromycin (Sigma), moxifloxacin, teicoplanin (Aventis), tetracycline (Sigma) and vancomycin (Lilly). Three additional drugs, oxacillin (Sigma), gentamicin (Sigma) and rifampicin (Sigma), were also tested against the Staphylococcus spp. Only AST results of 252 Gram-negative strains and 151 Gram-positive cocci (109 Staphylococcus spp. and 42 Enterococcus spp.) with correct IDs were compared with those of the MB method according to the National Committee for Clinical Laboratory Standards guidelines [9]. Minimum inhibitory concentration (MIC) agreement rates could show the accuracy of the Phoenix system. The rates were equal to the total number of strains minus the number of strains with the category agreement and discrepancy. The results were divided by the total number of strains tested and multiplied by $100 \%$.
Quality Control

Staphylococcus aureus American Type Culture Collection (ATCC 25923, ATCC 29213), Enterococcus faecalis(ATCC 29212), Escherichia coli (ATCC 25922, ATCC 35218, National Collection of Type Cultures NCTC 10418) and Pseudomonas aeruginosa (ATCC 27853, NCTC 10662) were incorporated as quality control strains. The ID and AST results of all quality control strains were confirmed correct before the clinical isolates were tested by the Phoenix system. If discrepant ID or AST results occurred, the strains were retested in duplicates by both methods.

\section{Results}

\section{Identification}

The Phoenix system gave reproducible IDs after comparing with those of the API ID system. Among 291 Gram-negative bacteria tested, 266 (91.4\%) were identified correctly to species level (table 1), while additional 18 organisms were correctly identified to genus level; thus, the system had correct ID rate of $97.6 \%$ (284 out of $291)$ to genus level. Hence, only 7 organisms (2.4\%) were misidentified (table 2). All 114 Staphylococcus spp. (100\%) tested, including 108 S. aureus, 2 S. capitis, 2 S. epidermidis, $1 S$. lugdunensis and $1 S$. warneri, were correctly identified to species level. Among 44 Enterococcus spp. tested, $93.2 \%$ were correctly identified to species level including 32 E. faecalis, 8 E. faecium and 1 E. avium. Two E. faecalis and one E. faecium strains were incorrectly identified by the Phoenix.

\section{Antimicrobial Susceptibility Testing}

The Phoenix system provided reproducible AST results after comparing with those of the MB method. For the 3,276 Gram-negative bacteria-antibiotic combinations, i.e. 252 strains tested against 13 antimicrobial agents, the agreement rates between the Phoenix MICs and the MB method MICs within \pm 1 dilution ranged from 87.3 to $97.6 \%$ (table 3a). Only $20(0.6 \%)$ and 4 $(0.1 \%)$ results had major or very major discrepancies, respectively. One hundred and nine Staphylococcus spp. tested against 11 antimicrobial agents had a total of 1,199 bacteria-antibiotic combinations. The agreement rates between the Phoenix MICs and the MB method MICs within \pm 1 dilution ranged from 54.6 to $100 \%$ (table 3 b). Only 4 out of 1,199 bacteria-antibiotic combinations $(0.3 \%)$ with major or very major discrepancies were found (table 3b). Three out of four major or very major discrepancies were found from oxacillin AST results (2 major and 1 very major discrepancies). For 336 bacteria-antibiotic combinations of the 42 Enterococcus spp., the agree- 
Table 1. The Gram-negative bacilli with correct identifications with the Phoenix system

\begin{tabular}{|c|c|}
\hline Organism name & Strains \\
\hline Acinetobacter baumannii & 9 \\
\hline Acinetobacter lwoffii & 1 \\
\hline Aeromonas caviae & 1 \\
\hline Aeromonas hydrophila & 1 \\
\hline Aeromonas veronii & 1 \\
\hline Agrobacterium radiobacter & 1 \\
\hline Burkholderia cepacia & 2 \\
\hline Chrysebacterium indologenes & 1 \\
\hline Chryseobacterium meningosepticum & 2 \\
\hline Citrobacter freundii & 4 \\
\hline Citrobacter koseri & 5 \\
\hline Enterobacter aerogenes & 9 \\
\hline Enterobacter cloacae & 8 \\
\hline Escherichia coli & 32 \\
\hline Klebsiella oxytoca & 5 \\
\hline Klebsiella pneumoniae & 31 \\
\hline Morganella morganii & 10 \\
\hline Ochrobactrum anthropi & 2 \\
\hline Proteus mirabilis & 16 \\
\hline Proteus vulgaris & 1 \\
\hline Pseudomonas aeruginosa & 67 \\
\hline Pseudomonas fluorescens & 1 \\
\hline Pseudomonas putida & 3 \\
\hline Pseudomonas stutzeri & 2 \\
\hline Providencia stuartii & 1 \\
\hline Salmonella paratyphi $A$ & 1 \\
\hline Salmonella spp. & 19 \\
\hline Salmonella typhi & 8 \\
\hline Serratia marcescens & 7 \\
\hline Stenotrophomonas maltophilia & 15 \\
\hline Total & 266 \\
\hline
\end{tabular}

Total number of Gram-negative strains tested $=291$.

The rate of correct identifications after comparing with the API system to species level $=266 / 291(91.4 \%)$.

Table 2. Gram-negative bacilli misidentified by the Phoenix system

\begin{tabular}{ll}
\hline Identity with the API system & Identity with the Phoenix system \\
\hline Alcaligenes xylosoxidans & $\begin{array}{l}\text { Pseudomonas sp. } \\
\text { Klebsiella pneumoniae ssp. } \\
\text { Ozaenae }\end{array}$ \\
Chryseomonas luteola & Sphingomonas paucimobilis \\
Enterobacter cloacae & Citrobacter freundii \\
Pantoea agglomerans & Enterobacter hormaechei \\
Pantoea sp. & Shigella flexneri \\
Serratia liquefaciens & Klebsiella pneumoniae ssp. \\
& ozaenae \\
\hline
\end{tabular}

ment rates between the Phoenix MICs and the MB method MICs within \pm 1 dilution ranged from 50.0 to $100 \%$ (table 3 c). Only 6 out of 336 combinations (1.8\%) had major or very major discrepancies. In both cases, vancomycin had low MIC agreement rates attributed to the high category agreement rates (interpretive category agreed, but MIC discrepancy $>1$ dilution), i.e. 45.4\% (49 out of 108) for Staphylococcus spp. and 50\% (21 out of 42) for Enterococcus spp. All vancomycin MICs of the Phoenix system were higher than those of the MB method in all vancomycin category agreement cases for both Staphylococcus spp. (49 out of 49) and Enterococcus spp. (21 out of 21).

\section{Discussion}

This study focused on the performance of the Phoenix Automated Microbiology System by testing Gram-negative bacilli and Gram-positive cocci commonly isolated in our routine clinical laboratory. The Phoenix system is composed of three major components including (a) a carousel-based panel transport system, (b) an optical reader capable of making both colorimetric and fluorometric measurements as a function of time and (c) a data analysis process. The reaction panels contain chemicals and indicators that yield visible absorption or fluorescence data for identifying the microorganisms. The principle of Phoenix AST is based on using oxidation-reduction reaction to monitor microbial metabolisms by the color changes of the indicator. If the organism is inhibited by an antimicrobial agent, there will be no dye reduction or color change. The instrument has the capacity holding 100 test panels. The results of the panel are read at 20min intervals by the instrument. The final interpretations of ID and AST results are available in $2-12 \mathrm{~h}$ and 4$12 \mathrm{~h}$, respectively.

According to an evaluation of the Automated Phoenix System by Donay et al. [10], the percentages of correct IDs were 93.3 and $89.4 \%$ for enterobacteria and nonfermenting Gram-negative bacilli, respectively, similar to our results. In our study, 5 out of 7 misidentified Gramnegative bacilli were nonfermenters (2 Pantoea spp., 2 Chryseomonas spp. and 1 Alcaligenes xylosocidans). The slower metabolism rates of nonfermenters caused weaker biochemical reactions in the reaction wells of the Phoenix panels, and therefore the ID results may be less reliable. The ID agreement rates of Staphylococcus spp. and Enterococcus spp. by Fahr et al. [8] were 97.1 and 98.9\%, also similar to our findings. The high ID agreement rates 
Table 3. Results of the antimicrobial susceptibility testing

\begin{tabular}{lllll}
\hline Antimicrobial agents & $\begin{array}{l}\text { MIC } \\
\text { agreement }^{\mathrm{a}}\end{array}$ & $\begin{array}{l}\text { Category } \\
\text { agreement }^{\mathrm{b}}\end{array}$ & $\begin{array}{l}\text { Discrepancy } \\
\text { minor }^{\mathrm{c}} \text { major }^{\mathrm{d}} \text { very major }^{\mathrm{e}}\end{array}$ \\
\hline
\end{tabular}

a Agreement of the broth microdilution method and the Phoenix system for 252 Gram-negative bacilli

\begin{tabular}{|c|c|c|c|c|c|}
\hline Amikacin & $236(94.8)$ & 10 & 3 & 0 & 3 \\
\hline Gentamicin & $231(90.8)$ & 9 & 5 & 6 & 1 \\
\hline Ampicillin-sulbactam & $245(97.2)$ & 3 & 4 & 0 & 0 \\
\hline Cefepime & $241(95.6)$ & 8 & 2 & 1 & 0 \\
\hline Cefotaxime & $238(94.4)$ & 2 & 11 & 1 & 0 \\
\hline Ceftazidime & $241(95.6)$ & 8 & 2 & 1 & 0 \\
\hline Chloramphenicol & $220(87.3)$ & 25 & 7 & 0 & 0 \\
\hline Ciprofloxacin & $245(97.2)$ & 6 & 0 & 1 & 0 \\
\hline Imipenem & $236(93.7)$ & 12 & 4 & 0 & 0 \\
\hline Meropenem & $246(97.6)$ & 6 & 0 & 0 & 0 \\
\hline Moxifloxacin $^{\mathrm{f}}$ & $233(93.2)$ & 13 & 4 & 0 & 0 \\
\hline Piperacillin & $221(87.7)$ & 11 & 12 & 8 & 0 \\
\hline Piperacillin-tazobactam & $223(88.5)$ & 23 & 4 & 2 & 0 \\
\hline
\end{tabular}

b Agreement rates of the Phoenix system vs. the broth microdilution method for 109 Staphylococcus spp.

\begin{tabular}{lccccc}
\hline Ampicillin & $109(100.0)$ & 0 & 0 & 0 & 0 \\
Chloramphenicol & $94(84.7)$ & 2 & 12 & 0 & 0 \\
Ciprofloxacin & $108(99.1)$ & 0 & 1 & 0 & 0 \\
Erythromycin & $97(89.8)$ & 5 & 6 & 0 & 0 \\
Gentamicin & $109(100)$ & 0 & 0 & 0 & 0 \\
Moxifloxacin & $102(93.6)$ & 7 & 0 & 0 & 0 \\
Oxacillin & $98(90.7)$ & 7 & 0 & 2 & 1 \\
Rifampicin & $108(100.0)$ & 0 & 0 & 1 & 0 \\
Teicoplanin & $89(82.4)$ & 19 & 0 & 0 & 0 \\
Tetracycline & $105(97.2)$ & 1 & 2 & 0 & 0 \\
Vancomycin & $59(54.6)$ & 49 & 0 & 0 & 0 \\
\hline
\end{tabular}

c The MIC agreement rates of the Phoenix system vs. the broth microdilution method for 42 Enterococcus spp.

\begin{tabular}{llllll}
\hline Ampicillin & $41(97.6)$ & 1 & 0 & 0 & 0 \\
Chloramphenicol & $34(81.0)$ & 0 & 5 & 3 & 0 \\
Ciprofloxacin & $32(80.0)$ & 0 & 5 & 3 & 0 \\
Erythromycin & $41(97.6)$ & 0 & 1 & 0 & 0 \\
Moxifloxacin & $40(95.2)$ & 1 & 1 & 0 & 0 \\
Teicoplanin & $42(100.0)$ & 0 & 0 & 0 & 0 \\
Tetracycline & $40(95.2)$ & 2 & 0 & 0 & 0 \\
Vancomycin & $21(50.0)$ & 21 & 0 & 0 & 0
\end{tabular}

Figures represent the number of strains. Percentages are given in the parentheses.

a Within \pm 1 dilution.

b Interpretive category agreement (MICs differed by $>1$ dilution).

c Susceptible or resistant with the Phoenix system and intermediate by the reference test or intermediate with the Phoenix system and susceptible or resistant by the reference test.

d Resistant with the Phoenix system and susceptible by the reference test.

e Susceptible with the Phoenix system and resistant by the reference test.

${ }^{f}$ No NCCLS breakpoint recommendation. 
between the Phoenix system and the API system of both Gram-negative bacilli and Gram-positive cocci showed that the Phoenix system is also a reliability ID system.

The overall AST category agreement rate of Gramnegative bacteria in our study is similar to that of Donay et al. [10]. The vancomycin MIC agreement rates of Staphylococcus spp. and Enterococcus spp. in the study by Fahr et al. [8] were 97.6 and $98.2 \%$, respectively; they are higher than our findings of $45.4 \%$ for Staphylococcus spp. and 50\% for Enterococcus spp. All vancomycin MICs of the Phoenix system were higher than those of the MB method in all vancomycin category agreed but not MICagreed cases for both Staphylococcus spp. and Enterococcus spp. A possible explanation may be that the readings of the final concentrations of the vancomycin in the reaction wells of the Phoenix PMIC/ID-14 panels were slightly but consistently lower. Hence, the vancomycin MICs of the Phoenix system were consistently higher than those of the MB method in all vancomycin category agreement cases for both Staphylococcus spp. and Enterococcus spp. Though only $0.3 \%$ (4 of 1,199 bacteria-antibiotic combinations) major or very major discrepancy was found for Staphylococcus spp., 3 of 4 discrepancies were found from the oxacillin AST results that were critical clinically. All discrepant AST results of vancomycin and oxacillin were repeated by both the Phoenix automated system and the
MB method for verification. This discrepancy requires further investigations.

As the Phoenix system is an open system, adding bacterial suspension to the testing panels manually may pose potential biohazard due to spillage. Sometimes, the panel covers may pop out in the incubation chamber. Despite these minor problems, the Phoenix system is an efficient instrument that can greatly decrease the turnaround and hand-on time of routine clinical laboratories.

\section{Conclusion}

The results indicate that the Phoenix system is a reliable system that could provide accurate ID and AST results for routine clinical laboratories.

\section{Acknowledgments}

We are grateful to the technical staff of the Department of Microbiology, The Prince of Wales Hospital, for their assistance. Part of this study has been presented at the 8th Western Pacific Congress on Chemotherapy and Infectious Diseases, 2002, Singapore. We would like to thank BD Company for providing the BD Phoenix Automated Microbiology System and its disposal panels.

\section{References}

$\checkmark 1$ Funke G, Monnet D, deBernardis C, von Graevenitz A, Freney J: Evaluation of the VITEK 2 system for rapid identification of medically relevant Gram-negative rods. J Clin Microbiol 1998;36:1948-1952.

-2 Jossart MF, Courcol RJ: Evaluation of an automated system for identification of Enterobacteriaceae and nonfermenting bacilli. Eur J Clin Microbiol Infect Dis 1999; 18:902-907.

-3 Ling TKW, Tam PC, Liu ZK, Cheng AFB: Evaluation of VITEK 2 rapid identification and susceptibility testing system against Gramnegative clinical isolates. J Clin Microbiol 2001;32:1757-1762.

$\checkmark 4$ Ligozzi M, Bernini C, Bonora MG, de Fatima M, Zuliani J, Fontana R: Evaluation of the Vitek 2 system for identification and antimicrobial susceptibility testing of medically relevant Gram-positive cocci. J Clin Microbiol 2002; 40:1681-1686. $\checkmark 5$ Endimiani A, Luzzaro F, Tamborini A, Lombardi G, Elia V, Belloni R, Toniolo A: Identification and antimicrobial susceptibility testing of clinical isolates of nonfermenting Gram-negative bacteria by the Phoenix Automated Microbiology System. New Microbiol 2002;25:323-329.

-6 Brisse S, Stefani S, Verhoef J, Van Belkum A, Vandamme P, Goessens W: Comparative evaluation of the BD Phoenix and VITEK 2 automated instruments for identification of isolates of the Burkholderia cepacia complex. J Clin Microbiol 2002;40:1743-1748.

7 Gavin PJ, Warren JR, Obias AA, Collins SM, Peterson LR: Evaluation of the Vitek 2 system for rapid identification of clinical isolates of Gram-negative bacilli and members of the family streptococcaceae. Eur J Clin Microbiol Infect Dis 2002;21:869-874.
$>8$ Fahr A, Eigner U, Armbrust M, Caganic A, Dettori G, Chezzi C, Bertoncini L, Benecchi M, Menozzi MG: Two-center collaborative evaluation of the performance of the BD Phoenix Automated Microbiology System for identification and antimicrobial susceptibility testing of Enterococcus spp. and Staphylococcus spp. J Clin Microbiol 2003;41:1135-1142.

9 National Committee for Clinical Laboratory Standards: Performance Standards for Antimicrobial Disk Susceptibility Tests. NCCLS document. Wayne, National Committee for Clinical Laboratory Standards, 2002, vol 20, issue 1.

10 Donay J-L, Mathieu D, Fernandes P, Pregermain C, Bruel P, Wargnier A, Casin I, Weill FX, Lagrange PH, Herrmann JL: Evaluation of the Automated Phoenix System for potential routine use in the clinical microbiology laboratory. J Clin Microbiol 2004;42:1542-1546. 